\title{
Limited evidence to recommend against open chest cardiopulmonary resuscitation in blunt trauma
}

\author{
Stefano Malinverni* ${ }^{*}$ and Pierre Mols
}

See related research by Endo et al., https://ccforum.biomedcentral.com/articles/10.1186/s13054-017-1759-1.

\section{Main Text}

We congratulate Endo and co-authors on their retrospective registry-based analysis comparing closed-chest cardiopulmonary resuscitation (CCCPR) and open-chest cardiopulmonary resuscitation (OCCPR) in cardiac arrest patients following blunt trauma [1] and would like to share the following comments.

OCCPR is generally deemed futile in blunt trauma patients whenever cardiopulmonary resuscitation (CPR) is ongoing for more than 10 minutes without the patient showing any signs of life [2]. Among patients for whom OCCPR is recommended expected survival doubles whenever the patient is in profound shock compared to patients without vital signs [2]. The lack of information about CPR duration and circulatory status before OCCPR initiation jeopardizes the interpretation of the overall results as great heterogeneity might exist between the two propensityscore matched groups. It is potentially misleading to draw conclusions about efficacy of a treatment (OCCPR) whenever indications of the aforementioned treatment cannot be assessed.

A further confounding element is that OCCPR is initiated in most of the cases for logistic reasons after initial treatment by CCCPR. During this initial window period a substantial proportion of potentially salvageable patients might achieve return over spontaneous circulation
(ROSC) [3]. Early ROSC is associated with increased survival [4], and it precludes any further treatment by OCCPR. Therefore, patients from this registry treated through OCCPR should be considered as having an intrinsically worse prognosis at treatment initiation despite rigorous stratification and matching.

The results would be more consistent if duration of CCCPR before OCCPR were known. This would allow exclusion from the analysis of cases where ROSC occurred before the average duration of CCCPR at OCCPR initiation.

While acknowledging that the utilization of an instrumental variable (IV) might be used to control for unmeasurable confounders in non-randomized experiments, its choice should nevertheless respect the fundamental assumption that the IV is not directly associated with the outcome variable [5]. The IV "mean number of OCCPR cases per year in the hospital" captures hospital characteristics in a given year, which can be directly linked to survival as high-volume centers in terms of OCCPR are probably associated with increased resuscitation skills through this technique. This invalidates the use of the IV to measure OCCPR implementation.

In our opinion OCCPR in blunt trauma cannot be rigorously associated with reduced rates of hospital discharge and of 24-h survival following Emergency Department admission based on the available evidence.

\section{Authors' response}

\section{Akira Endo, Atsushi Shiraishi and Yasuhiro Otomo}

We would like to thank Malinverni et al. for their comments regarding our recent article [1]. We had to

\footnotetext{
* Correspondence: stefano_MALINVERNI@stpierre-bru.be

Emergency Department, Centre Hospitalier Universitaire Saint Pierre, Université Libre de Bruxelles, Rue Haute 322, 1000 Brussels, Belgium
}

compromise on the study design as a retrospective observational study since this research question could not be evaluated by a randomized controlled trial due to ethical issues. Although we adjusted for the biases as much as possible using the best conceivable statistical 
models for causal inferences, several unsolvable problems remained due to the study design.

Information on the duration of CCCPR before the implementation of OCCPR was lacking in the Japan Trauma Data Bank, and could be considered as an unmeasured confounder. However, we performed subgroup analysis according to the timings of cardiac arrest (at the scene of injury and upon arrival at the emergency department) and pre-hospital transport time, which were considered to be greatly associated with CCCPR duration before OCCPR, and could be surrogate variables. Interestingly, these subgroup analyses did not show significant interactions and did not have any trends for favorable/unfavorable outcomes, indirectly suggesting that the impact of CCCPR duration before OCCPR on in-hospital survival was limited. As for the concern regarding the IV we used, to our knowledge, the volumeoutcome relationships regarding OCCPR have not been reported previously, and these relationships do not always exist in surgical procedures [6]. It is considered to be insufficient to deny eligibility of the IV based on unfounded assumptions. At least, it can be said that our research failed to show the effectiveness of OCCPR in multiple models adjusted as much as possible for measured/unmeasured biases.

We would like to emphasize that we did not evaluate the effectiveness of emergency department thoracotomy (EDT), but only a procedure of OCCPR that is a part of EDT, which includes hemostasis of intra-thoracic organs and/or aortic cross-clumping. EDT is occasionally also performed in patients without cardiac arrest. Several reports have described cases where patient survival was dependent on EDT including OCCPR [7]; we have also experienced such cases.

However, we should recognize that the evidence supporting the effectiveness of OCCPR remains scarce, at least among blunt trauma patients as a whole, and we have to conduct further studies to assess a limited specific population who benefit from EDT including OCCPR.

\section{Abbreviations}

CCCPR: Closed-chest cardiopulmonary resuscitation; CPR: Cardiopulmonary resuscitation; EDT: Emergency department thoracotomy; IV: Instrumental variable; OCCPR: Open-chest cardiopulmonary resuscitation; ROSC: Return over spontaneous circulation

\section{Acknowledgements}

None to report.

\section{Funding}

No funding to report.
Authors' contributions

SM and PM both contributed equally in writing the manuscript. Both authors read and approved the final manuscript.

Authors' information

None.

Ethics approval and consent to participate

Not applicable.

Consent for publication

Not applicable.

\section{Competing interests}

The authors declare that they have no competing interests.

\section{Publisher's Note}

Springer Nature remains neutral with regard to jurisdictional claims in published maps and institutional affiliations.

Published online: 20 September 2017

\section{References}

1. Endo A, Shiraishi A, Otomo Y, Tomita M, Matsui H, Murata K. Open-chest versus closed-chest cardiopulmonary resuscitation in blunt trauma: analysis of a nationwide trauma registry. Crit Care. 2017:21(1):169.

2. Burlew CC, Moore EE, Moore FA, Coimbra R, McIntyre Jr RC, Davis JW, et al. Western Trauma Association critical decisions in trauma: resuscitative thoracotomy. J Trauma Acute Care Surg. 2012;73:1359-63.

3. Goto Y, Funada A, Goto Y. Relationship between the duration of cardiopulmonary resuscitation and favorable neurological outcomes after out-of-hospital cardiac arrest: a prospective, nationwide, population-based cohort study. J Am Heart Assoc. 2016;5(3):e002819. doi:10.1161/JAHA.115. 002819

4. Rajan S, Folke F, Kragholm K, Hansen CM, Granger CB, Hansen SM. Prolonged cardiopulmonary resuscitation and outcomes after out-ofhospital cardiac arrest. Resuscitation. 2016:105:45-51.

5. Rassen JA, Schneeweiss S, Glynn RJ, Mittleman MA, Brookhart MA. Instrumental variable analysis for estimation of treatment effects with dichotomous outcomes. Am J Epidemiol. 2009;169:273-84.

6. Kozower BD, Stukenborg GJ. Volume-Outcome Relationships in Thoracic Surgery. Thorac Surg Clin. 2017;27:251-6.

7. Hamidian Jahromi A, Northcutt A, Youssef AM. A Patient With Blunt Trauma and Cardiac Arrest Arriving Pulseless at the Emergency Department; is that Enough Reason to Stop Resuscitation? Review of Literature and Case Report. Iran Red Crescent Med J. 2013;15:e11623. 